\title{
Time-frequency and network analysis of hippocampus in APP/PS1 double transgenic mice with dementia of different degrees
}

\author{
Duyan Geng ${ }^{1,2}$, Zeyu $\mathrm{Gao}^{3}$, Yan Wang ${ }^{2}$, Zhaoxu Qin ${ }^{3}$, Geng Pang ${ }^{3}$, Ning Zhao ${ }^{3}$ \\ ${ }^{1}$ State Key Laboratory of Reliability and Intelligence of Electrical Equipment, Hebei University of \\ Technology, Tianjin 300130, China \\ ${ }^{2}$ School of Electrical Engineering, Hebei University of Technology, Tianjin 300130, China \\ ${ }^{3}$ School of Health Sciences\&Biomedical Engineering, Hebei University of Technology, Tianjin \\ 300130, China
}

Received: June 28, 2021. Revised: December 8, 2021. Accepted: January 12, 2022. Published: January 13, 2022.

\begin{abstract}
Hippocampal atrophy and neuron loss are common symptoms of Alzheimer's disease (AD). The hippocampal region is well known for producing oscillations at different frequency bands due to the neuronal network architecture. However, the mechanism of Ripple high frequency variation in hippocampal region with the course of AD disease has not been correctly assessed. We proposed time-frequency analysis using wavelet transform and constructing Granger causality network to analyze the characteristics of Hippocampal sharp wave-ripple (SPW-R) complexes in APP/PS1 mice at different cognitive levels. We use wavelet transform to overcome the shortcoming that the traditional Short Time Fourier Transform cannot deal with the unsteady signal frequency, and construct the Granger causality network to verify our results. By analyzing ripple frequency band energy changes and directional transfer function matrix in hippocampal CA1 region of mice with different cognitive levels, we found that the loss of ripple high frequency energy and decreased network connectivity in hippocampal CA1 region of APP/PS1 mice were correlated with the degree of memory loss. We believe that from mild dementia to severe dementia. The decreased cell activity in APP/PS1 mouse CA1 region leads to changes in Ripple high-frequency time-frequency energy and network connectivity for theoretical reasons. Our results provide support for assessing cognitive loss in APP/PS1 mice from the perspective of Ripple high frequency in hippocampus CA1 region.
\end{abstract}

Keywords - Alzheimer's disease, Hippocampal, Sharp wave-ripple, Time-frequency analysis, Granger causality network

\section{INTRODUCTION}

A lzheimer's disease (AD) is the most common degenerative central nervous system disease in the elderly. The pathologic cause of $A D$ is thought to be amyloid beta $(A \beta)$ in the hippocampus and senile plaques (SP) formed by neurofibrillary tangles (NFT) composed of phosphorylated Tau protein [1], which leads to decreased cell activity in the hippocampus [2]. However, there are still many uncertainties in the pathogenesis of $\mathrm{AD}[3], \mathrm{AD}$ is characterized by progressive memory impairments, language, behavioral, visuospatial impairment, and personality changes [4]. The medical diagnosis of Alzheimer's disease is difficult, especially in the early stages of the disease, mainly because symptoms are often ignored as a normal consequenc

e of aging [5]. If the method of genomics is used to identify diseases, it has a very high accuracy, but this method is expensive and requires the construction of different gene pools according to race [6]. The method of electrophysiology can overcome these difficulties.

The hippocampal neuronal network architecture is ideally suited to provide the framework for generating slow and fast oscillations ranging from very slow to ultra-fast $(0.025-600 \mathrm{~Hz})$ [7]. The oscillations in various frequency bands are associated to different behavioral states. Immobility and slow-wave sleep are associated with SPW-R complexes $(90-200 \mathrm{~Hz})$ [8-9]. This rhythm is largely confined to the hippocampal network, and recent evidence suggests that sharp ripples play a key role in some aspects of active spatial navigation [10]. Studies on the field potential in the hippocampus and cerebral cortex show that the Ripple high frequency oscillation is also related to the 
process of information transmission from the hippocampus to the cerebral cortex [11]. Several studies indicate that interneurons and consequently the oscillatory network activities regulated by them are altered in AD [12]. However, the mechanisms by which oscillating network activity varies with the degree of $\mathrm{AD}$ disease progression are poorly understood.

Time-frequency analysis is to Transform the time-domain signal into time-domain and frequency-domain analysis. Wavelet Transform (WT) is a common method for non-stationary signal processing and analysis. WT overcomes the shortcoming that the resolution of short-time Fourier Transform is constrained by window functions, and replaces the infinite length trigonometric function basis of Fourier Transform with the attenuation wavelet basis of finite length, whose basis function forms time-frequency Windows of various widths after stretching and translation [13]. replaces the infinite length trigonometric function basis in the Fourier transform with the attenuation wavelet basis of finite length, whose basis function forms time-frequency Windows of various widths after stretching and translation. When the signal frequency is low, THE time window of WT is wide and the frequency window is narrow; when the signal frequency is high, the time window of WT is narrow and the frequency window is wide. Therefore, WT has variable resolution and can adaptively analyze signals of different time scales.

Based on the comparison of model errors, Granger causality analysis can analyze the causal relationship between multiple time variables, which means that it is applicable to all kinds of random signals and therefore can be used to reveal connectivity patterns in neuroscience research. Therefore, it has been widely used in the field of neural signals [14]. Granger causality analysis can reflect the state of directional connections between brain activities recorded during a specific task [15]. In recent years, new causal analysis methods such as Direct Transfer Function (DTF) and Partial Directed Coherence (PDC) have been developed based on granger causality analysis.

Therefore, this study aims to analyze potential changes between Ripple high frequency and cognitive loss in APP/PS1 mice through time-frequency analysis and construction of Granger causality networks. We used the Morris water maze test to differentiate the cognitive level of multi-month-old APP/PS1 mice. We used Morris water maze test to distinguish the cognitive level of APP/PS1 mice aged several months. Considering that the cognitive level of mice will decline with the increase of age, we also selected C57BL/6J wild type mice of the same age as mice with different dementia levels as the control group, so as to judge the influence of age on the cognitive level of mice and eliminate the errors caused by age. Then, chronic electrode implantation was performed on the hippocampal CA1 region of mice, and the resting state LFP was collected after good recovery. We comparing and analyzing the energy and network differences of neural electrophysiological signals between different groups. Grouping APP/PS1 mice with different cognitive levels and C57 mice of corresponding age, we studied the characteristics of hippocampal SPW-R monitored by multi-channel in vivo implantation technique.
Our results played a positive role in the study of the potential changes of ripple frequency and degree of cognitive loss in hippocampal CA1 region of APP/PS1 mice, and had certain reference value. The results indicated that the network activity of APP/PS1 mice with different cognitive levels was significantly different compared with that of mice.

\section{MATERIAL AND METHODS}

\section{A. Animal}

A well-established and extensively utilized animal model for $\mathrm{AD}$ is the double transgenic APP/PS1 mouse which overexpresses familial AD mutations of human amyloid precursor protein and presenilin-1, resulting in increased $A \beta 42$ levels and thus representing a model of cerebral amyloidosis for AD, with early onset of the amyloid plaque deposition [16]. The APP/PS1 double transgenic mice and C57 mice were purchased from Beijing Huafukang Biotechnology Co, Ltd. All mice were kept in a SPF animal laboratory at $(25 \pm 2){ }^{\circ} \mathrm{C}$ and $(75 \pm 5) \%$ humidity. They were housed in a single cage with 12 hours of light and 12 hours of darkness every day. The mice were fed with normal breeding materials and had free access to food and water. The model showed that amyloid plaques began to deposit in the neocortex at 2 months of age and in the hippocampus at 4-5 months of age [17]. The mice were divided into 5, 8and 12 months old according to age, in which APP/PS1 mice were the $\mathrm{AD}$ group, and $\mathrm{C} 57 \mathrm{BL} / 6 \mathrm{~J}$ mice were the $\mathrm{C} 57$ group for control, with 10 mice in each group. The procedures for this study have been approved by the Biomedical Ethics Committee of Hebei University of Technology (review number: HEBUTaCUC2020003).

\section{B. Behavior}

Morris Water Maze (MWM) behavioral tests mainly test the ability of learning and remembering spatial location and orientation in experimental animals [18]. MWM was used to test the cognitive abilities of AD mice aged 5 months, 8 months and 12 months. In this study, the positioning navigation experiment commonly used in MWM experiment was used to distinguish the cognitive ability of mice [19]. The weaker the cognitive ability is, the more serious the disease is. The healthy mice of the same age were used as the control group. The final score of each mouse in the AD group was the average of its 6-day test score, and the ratio of the difference between this value and the reference value to the final score of the mouse was calculated. If the proportion was $>20 \%$, the mouse was considered to be demented; mild dementia was defined when the proportion was between 20 and $30 \%$, moderate dementia was defined when the proportion was $30-40 \%$, severe dementia was when the proportion was $>40 \%$.

\section{Electrophysiology}

We used a 16-channel microelectrode array $(4 \times 4,16$ contacting sites spaced by $200 \mu \mathrm{m}$, Plexon, America) to collect electrical activity signals in the CA1 region. A 128-channel neuroelectrophysiological signal acquisition system (Omni Plex, Plexon, America) was used to collect the raw data of 16-channel LFPS signals at rest in mice with different dementia 
degrees and control groups.

\section{Wavelet Transform}

We used wavelet transform to conduct time-frequency analysis on pretreated LFPs to study the variation characteristics of LFPs energy in time domain and frequency domain of mice with different dementia degrees. $\boldsymbol{\psi}(\boldsymbol{t}) \in \boldsymbol{L} \mathbf{2}(\boldsymbol{R})$, $\psi(t)$ can be obtained by FT transformation, if the $\psi(\omega)$ satisfies the following allowable conditions:

$$
C_{\psi}=\int_{0}^{+\infty} \frac{|\psi(\omega)|^{2}}{\omega} \mathrm{d} \omega<+\infty
$$

In the formula, $\boldsymbol{C} \boldsymbol{\psi}$ is the admissibility constant, and $\boldsymbol{\psi}(\boldsymbol{t})$ is the wavelet function. The wavelet basis function $\psi_{a}, \tau(t)$ can be obtained by translating and scaling the wavelet function. The process can be expressed as:

$$
\psi_{a, \tau}(t)=\frac{1}{\sqrt{a}} \psi\left(\frac{t-\tau}{a}\right), \quad a>0, \tau \in R
$$

$\boldsymbol{a}$ is a scaling function, which calibrates the analysis frequency of wavelet transform, $\tau$ is a time shift parameter, which defines the time of analysis, and $\Psi(t)$ is the mother wavelet.

$$
\alpha=\frac{2 \mathrm{nF}_{\mathrm{C}}}{\mathrm{k}}(\mathrm{k}=1,2 \cdots, \mathrm{n})(3)
$$

$\boldsymbol{F}_{\boldsymbol{C}}$ is the center frequency of the mother wavelet, $\boldsymbol{n}$ is the frequency division, and $\boldsymbol{K}$ is the normalized frequency. According to the experimental requirements, the mother wavelet is selected as CMOR3-3 [20], its center frequency $\boldsymbol{F}_{\boldsymbol{C}}=\mathbf{3}$, and the frequency division $\mathbf{N}=\mathbf{2 0 0 0}$ is selected, that is, the frequency resolution of $0.25 \mathrm{~Hz}$ is obtained for the signal with the sampling rate $\mathbf{F s}=\mathbf{1 0 0 0 H z}$

Where, $\boldsymbol{n}$ is the frequency division, $\boldsymbol{F}_{\boldsymbol{C}}$ is the center frequency of the mother wavelet, and $\boldsymbol{k}$ is the normalized frequency. Let $f(t) \in \boldsymbol{L} 2(R), \boldsymbol{\psi}(\boldsymbol{t})$ be a wavelet function, if the following conditions are satisfied:

$(W f)(a, \tau)=\left\langle f(t), \psi_{a, \tau}(t)\right\rangle=\frac{1}{\sqrt{a}} \int_{-\infty}^{+\infty} f(t) \bar{\psi}\left(\frac{t-\tau}{a}\right) \mathrm{d} t, \quad a>0, \tau \in R$

So, $(\boldsymbol{W} \boldsymbol{f})(\boldsymbol{a}, \boldsymbol{\tau})$ makes $\boldsymbol{f}(\boldsymbol{t})$ Continuous Wavelet Transform (CWT). Therefore, wavelet transform is a continuous integral transform, and can transform a univariate function into a binary function. When the following conditions are met:

$$
f(t)=\frac{1}{C_{\psi}} \int_{0}^{+\infty} \int_{-\infty}^{+\infty} \frac{1}{a^{2}}(W f)(a, \tau) \psi_{a, \tau}(t) \mathrm{d} \tau \mathrm{d} a
$$

It is said to be capable of reconstruction or contravariant transformation.

\section{E. Granger causality analysis}

DTF connection matrix of LFP-Ripple in hippocampus CA1 region of mice with different dementia degree was calculated, and LFP-Ripple causal network was constructed by directional transfer function method. Directional transfer functions (MVAR) were used to quantitatively describe the functional connectivity intensity between multichannel LFP. $\boldsymbol{X}(\boldsymbol{t})$ is LFP, and $\mathrm{N}$ is the number of channels:

$$
X(t)=\left\{\mathrm{x}_{1}(t), \mathrm{x}_{2}(\mathrm{t}) \ldots \mathrm{x}_{3}(t)\right\}
$$

Build the MVAR model:

$$
X(t)=\sum_{n=1}^{p} A_{n} X(t-n)+E(t)
$$

$\boldsymbol{A}_{\boldsymbol{n}}$ is the coefficient matrix of the MVAR model, and P is the order of the model, which can be determined by Bayesian Information Criterion (BIC) calculation. $\boldsymbol{E}(\boldsymbol{t})$ is the multivariable zero-mean non-correlated white noise matrix.

Convert the MVAR model to the frequency domain

$$
\begin{gathered}
X(f)=A^{-1}(f) E(f)=H(f) E(f) \\
H(f)=A^{-1}(f)=\left(\sum_{i=0}^{p} A(i) e^{-j 2 \pi f i \Delta t}\right)^{-1}
\end{gathered}
$$

$\boldsymbol{H}(\boldsymbol{f})$ is the transfer function matrix, $\boldsymbol{A}(\boldsymbol{\theta})=-\boldsymbol{I}$, and $\boldsymbol{I}$ is the identity matrix.

$\gamma_{i j}{ }^{2}$ is the causal connection value from channel $\boldsymbol{j}$ to channel $i$.

$$
\gamma_{\mathrm{ij}}(f)=\left|H_{\mathrm{ij}}\right|^{2} / \sum_{\mathrm{n}=1}^{N}\left|H_{i n}(f)\right|^{2}
$$

$\gamma_{i j}$ is the normalization of the proportion of channel $\boldsymbol{j}$ influence on channel $\boldsymbol{i}$ among all channels' influence on channel $\boldsymbol{i}$. The larger $\gamma_{i j}$ is, the str onger the c ausal connection between channel $\boldsymbol{j}$ and channel $\boldsymbol{i}$ is, and the weaker the causal connection is $\sum_{\mathrm{n}=1}^{N}\left|H_{\text {in }}(f)\right|^{2}$. Let $\boldsymbol{D} \boldsymbol{T} \boldsymbol{F}_{i j}=\gamma_{i j}$, and DTF matrix is formed by $D_{T F}$.

\section{RESULTS}

\section{A. Group}

In order to subdivide the degree of dementia in the model mice, we conducted MWM experiment on purchased APP/PS1 model mice aged 5, 8 and 12 months. According to the escape latency period of each model mouse and the age of the mouse, the mild dementia mice in the 5-month-old model mice were selected as the mild dementia experimental group. 5 months C57 mice as the control group, 8 months of age in the model mice with mild dementia mice as the mild dementia group, 8 months C57 mice as the control group, select 12 months the mild dementia mice as mild dementia model mice group, 12 months C57 mice as the control group, six groups of experimental animals, respectively in each group 8 .

\section{B. Time-frequency analysis}

Raw data of 16 channel LFP signal at rest in mice with different dementia degrees and control group. Three sections of resting state data were taken for each animal at different time. Data were taken from each mouse at rest for 10 seconds. The energy density distribution of Ripple high-frequency oscillations is shown in Figure 1, respectively for groups with different dementia degrees and the control group with the same age. The horizontal axis is group, and the vertical axis is ripple high frequency power peak. Color represents power from low 
(blue) to high (yellow).
MILD DEMENTIA

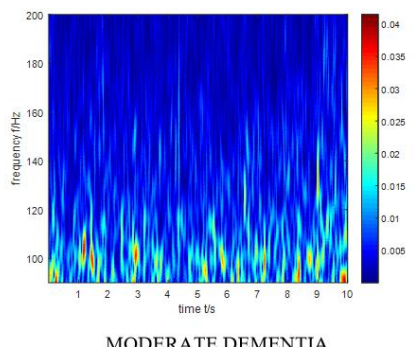

MODERATE DEMENTIA

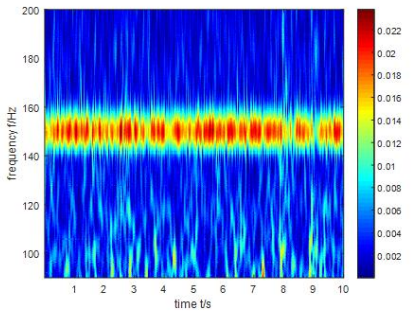

SEVERE DEMENTIA

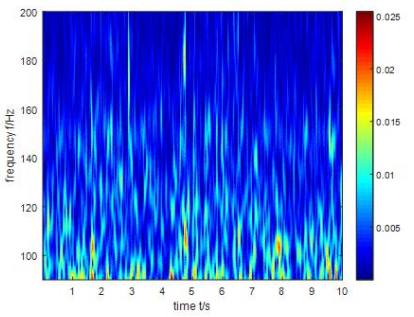

WT

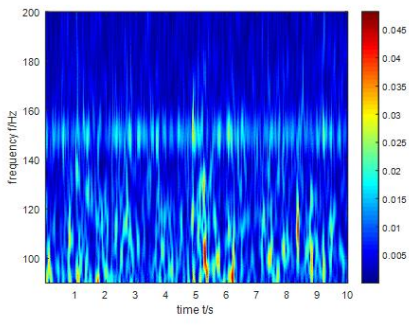

WT

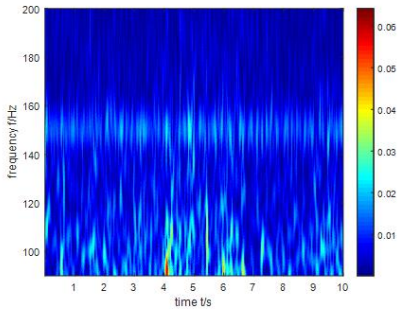

WT

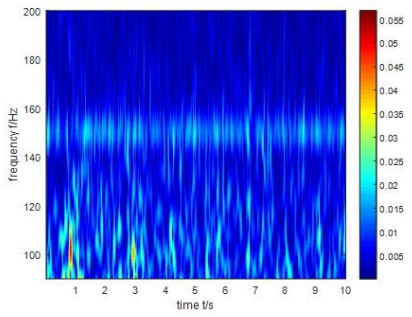

Fig. 1. Distribution of power of ripple high frequency band

Figure 2 represents the distribution ranges of Ripple high frequency oscillation energy density in groups with different dementia degrees and the control group at the same age. It can be seen from Figure 2 that the Ripple high frequency oscillation power of model mice with different dementia levels is significantly reduced in age compared with the control group $(\mathrm{P}<0.001)$, and energy loss were more severe with dementia. As can be seen from Figure 2, compared with C57 mice of the same age, the pulsation high-frequency oscillation power of mild dementia mice was lower than that of the control group, but not significantly. The pulsation high-frequency oscillation power of moderate dementia and severe dementia mice was significantly lower than that of the control group $(\mathrm{P}<0.001)$, energy loss was associated with greater dementia severity. As shown in Figure 2, ripple impact energy in CA1 region of mice in the control group showed no significant difference at the same time. In APP/ PS1 mice aged 5, 8 and 12 months, the effect of age on the ripple energy in the CA1 region of model mice was negligible. In APP/PS 1 mice aged 5, 8 and 12 months, Ripple oscillation energy in CA1 region seems to be more useful in determining dementia.
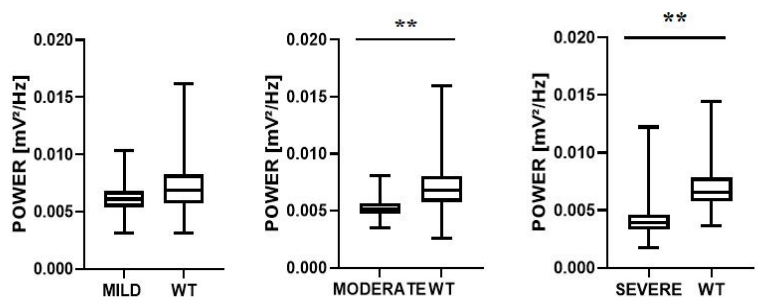

Fig. 2. Comparison of Ripple high-frequency oscillation energy density distribution interval

\section{Network connectivity analysis}

DTF connection matrix of 16 channel networks in hippocampal CA1 region of normal control mice and model mice with different dementia degrees at rest is shown in Figure 3. $\boldsymbol{D T F}_{i j}$ connection matrix can reflect the connection relationship between any node and other nodes. The color represents $\boldsymbol{D} \boldsymbol{T} \boldsymbol{F}_{\boldsymbol{i j}}$, representing the $\boldsymbol{D} \boldsymbol{T} \boldsymbol{F}_{\boldsymbol{i j}}$ maximum value (red) of the connection strength from channel $\mathrm{J}$ to channel $\mathrm{I}$, which is normalized to 1 , and the $\boldsymbol{D} \boldsymbol{T} \boldsymbol{F}_{i j}$ minimum value (blue), which is normalized to 0 .
MILD DEMENTIA

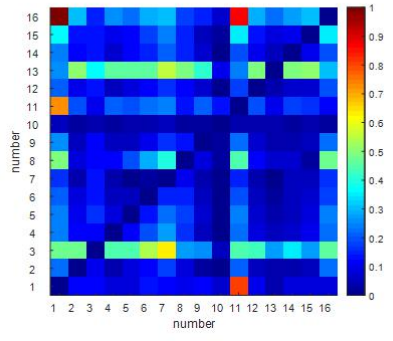

MODERATE DEMENTIA

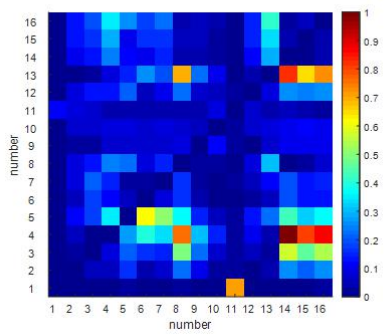

SEVERE DEMENTIA

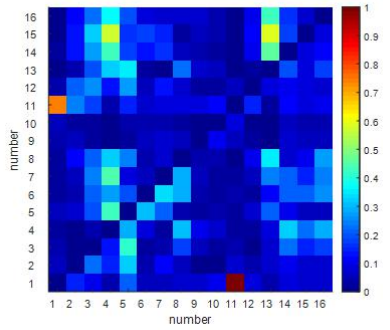

WT

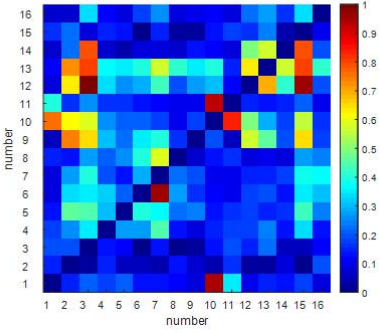

WT

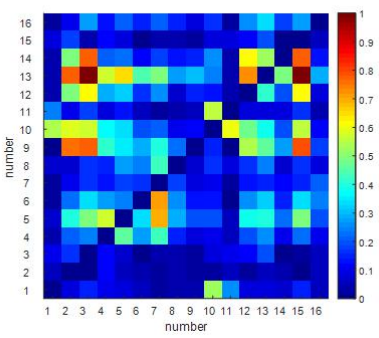

WT

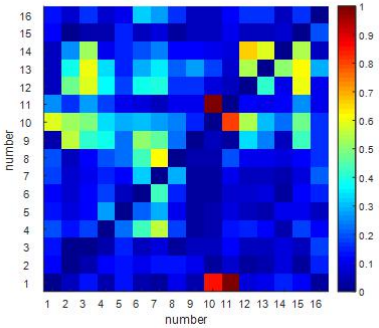

Fig. 3. DTF connection matrix of ripple high frequency band

Figure 4 shows the distribution range of DFT at the same age of the group with different dementia degrees and the control group. As can be seen from Figure 4, the average DTF value of 
granger causality network of model group mice compared with control group mice decreased significantly to different degrees from mild dementia to severe dementia. These findings suggest that network connectivity in model mice decreases with the onset of dementia. According to the findings of our from mild dementia, mild dementia and severe dementia model mice comparing DTF value that, with dementia degree deepening, DTF value model mice significantly decline, this phenomenon shows that since the degree of dementia mice hippocampal CA1 area of corrugated oscillation network connectivity significantly decline, whether for the analysis of the influence from the change of the age, We analyzed the DFT distribution of normal mice of different months, and found that the connectivity of ripple oscillation network in the hippocampal CA1 region of mice decreased with the increase of the age of mice. This phenomenon indicated that the influence of age on the connectivity of ripple oscillation network in the hippocampal CA1 region of mice could not be ignored. To determine whether the observed decrease was mainly due to changes in dementia degree or age, we analyzed the data and found that increased dementia degree had a stronger effect on decreased hippocampal CA1 network connectivity than age.
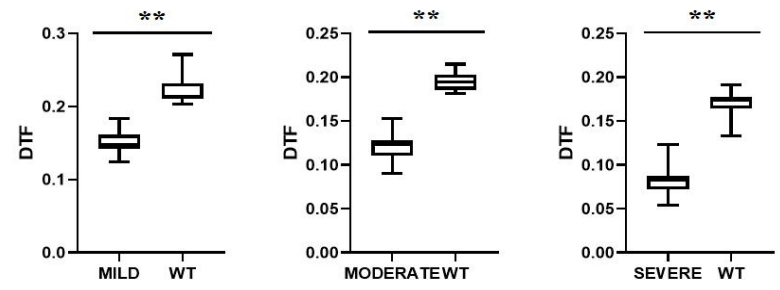

Fig. 4 Comparison of DTF connection matrix and average DTF of ripple high frequency band

\section{CONCLUSION}

$\mathrm{AD}$ is a progressive neurodegenerative disease, and cognitive decline is one of its main characteristics [3]. The APP/PS1 double transgenic mice were used as model to study $\mathrm{AD}$. In order to explore the extent of the condition in $\mathrm{AD}$ mice, the MWM experiment was used to detect the cognitive abilities of mice.

In this study, we attempt to evaluate the degree of dementia in model mice from the perspective of oscillation energy and network connectivity of ripple band in hippocampal CA1 region by using wavelet transform and Granger causality analysis. In particular, we use wavelet transform to overcome the disadvantage that traditional STFT cannot deal with unsteady signal frequencies. And construct Granger causality network to verify our results.

Hippocampal CA1 neurons are known to highly interconnect with one another, They are considered to play a key role in triggering and maintaining memory-related network activity patterns such as SPW-R in the hippocampus and other brain regions [21-22]. This has been widely demonstrated. Our study showed that cell activity and network connectivity in the CA1 region of APP/PS1 mice were reduced after mild dementia. This finding supports that neurons are severely damaged during the formation of amyloid plaques in the process of $\mathrm{AD}$ amyloidosis, resulting in damage to the connections between neurons [23]. Interestingly, we also observed that the ripple energy in hippocampal CA1 region of normal mice was less affected by time compared with the model group.

Our study showed that cell activity and network connectivity in the CA1 region of APP/PS1 mice were reduced after mild dementia. This is consistent with recent studies showing that $\mathrm{AD}$ causes age-related neuron loss. We compared the results in APP/PS1 mice with autopsy results in patients with $\mathrm{AD}$, which was consistent with previous reports of post-mortem brain neuron loss in patients with AD [24]. Similarly, we also observed that compared with the model group, the connectivity of the Ripple frequency network in hippocampal CA1 region of normal mice was also affected by time, which was consistent with the general situation that the hippocampal region aging showed decreased brain connectivity with the increase of age. However, it remains to be confirmed whether the same loss rate of network connectivity in hippocampal CA1 region exists in normal control mice and model mice.

These studies clearly indicate that ripple oscillatory activity is impaired in the hippocampus of $\mathrm{AD}$ mouse model and is closely related to cognitive impairment, which provides a new idea for the diagnosis of AD. Although similar studies in human AD patients have been very limited, it remains a legitimate question whether hippocampal neuronal activity can be safely and effectively restored in mice. If safe and effective methods can be found, such as obtaining a reasonable and effective frequency of TMS to restore hippocampal neuron function, it will provide another promising treatment for AD. However, it should be borne in mind that caution should be exercised when associating the mouse APP/PS1 phenotype with delayed AD in humans in any such animal model, most of which occurs in the absence of APP or PS1 mutations.

\section{References}

[1] DeTure, Michael A, and Dennis W Dickson. "The neuropathological diagnosis of Alzheimer's disease." Molecular neurodegeneration vol. 14, no.1, pp. 32, Aug. 2019.

[2] Zallo, F, Gardenal, E, Verkhratsky, A, \& Rodríguez, J. J."Loss of calretinin and parvalbumin positive interneurones in the hippocampal CA1 of aged Alzheimer's disease mice." Neuroscience letters vol. 681, no. 10, pp. 19-25.Aug. 2018.

[3] Bateman RJ, Xiong C, Benzinger TL, et al. "Clinical and biomarker changes in dominantly inherited Alzheimer's disease" The New England journal of medicine vol. 367, no. 9, pp. 795-804, Aug. 2012.

[4] Cedernaes J, Osorio RS, Varga AW, Kam K, Schiöth HB, Benedict C."Candidate mechanisms underlying the association between sleep-wake disruptions and Alzheimer's disease." Sleep medicine reviews vol. 31, no. 1 pp. 102-111, Feb. 2017. 
[5] Houmani N, Vialatte F, Gallego-Jutglà E, et al. "Diagnosis of Alzheimer's disease with Electroencephalography in a differential framework." PLoS One. Vol. 13, no.3, Mar. 2018.

[6] HIMA VYSHNAVI A M , P K KRISHNAN NAMBOORI "Identification of Lapatinib Derivatives and Analogs to Control Metastatic Breast Cancer-specific to South Asian Population-a Pharmacogenomic Approach" WSEAS TRANSACTIONS on BIOLOGY and BIOMEDICINE, vol. 18, pp.51-62, Apr 2021.

[7] T Klausberger, P Somogyi, "Neuronal diversity and temporal dynamics: the unity of hippocampal circuit operations." Science, vol 321, pp. 53-57.Jul. 2008.

[8] G Buzsáki, "Hippocampal sharp waves: their origin and significance.” Brain research, vol 398, pp. 242-252. Nov. 1986.

[9] Roumis DK, Frank LM. "Hippocampal sharp-wave ripples in waking and sleeping states." Curr Opin Neurobiol. Vol. 35, pp. 6-12. Dec. 2015.

[10] Jura B, Macrez N, Meyrand P, et al. "Deficit in hippocampal ripples does not preclude spatial memory formation in APP/PS1 mice". Scientific reports,vol. 9, no.1, Dec. 2019.

[11] Benthem SD, Skelin I, Moseley SC, Stimmell AC, Dixon JR, Melilli AS, Molina L, McNaughton BL, Wilber AA. "Impaired Hippocampal-Cortical Interactions during Sleep in a Mouse Model of Alzheimer's Disease." Current biology : CB,vol. 30, no.13, pp.2588-2601, Jul. 2020.

[12]Palop JJ, Mucke L. "Network abnormalities and interneuron dysfunction in Alzheimer disease." Nat Rev Neurosci. vol. 17, no.12, pp. 777-792, Dec. 2016.

[13] Rai H M , Trivedi A, Shukla S . "ECG signal processing for abnormalities detection using multi-resolution wavelet transform and Artificial Neural Network classifier". Measurement Journal of the International Measurement Confederation, vol. 46, no.9, pp. 3238-3246, Nov. 2013.

[14]K. Tripanpitak, S. He, I. Sönmezışık, T. Morant, S. Y. Huang and W. Yu, "Granger Causality-Based Pain Classification Using EEG Evoked by Electrical Stimulation Targeting Nociceptive A $\delta$ and C Fibers." in IEEE Access, vol. 9, pp. 10089-10106 ,Jan. 2021.

[15] Cekic S, Grandjean D, Renaud O. "Time, frequency, and time-varying Granger-causality measures in neuroscience." Statistics in medicine,vol. 37,no. 11,pp. 1910-1931,Mar. 2018.

[16] Van Hoesen GW, Hyman BT, Damasio AR. "Entorhinal cortex pathology in Alzheimer's disease". Hippocampus.vol. 1, no. 1, pp. 1-8, Jan. 1991.

[17] Radde R, Bolmont T, Kaeser SA, Coomaraswamy J, Lindau D, Stoltze L, Calhoun ME, Jäggi F, Wolburg H, Gengler S, Haass C, Ghetti B, Czech C, Hölscher C, Mathews PM, Jucker M. "Abeta42-driven cerebral amyloidosis in transgenic mice reveals early and robust pathology." EMBO Rep. vol. 7, no. 9, pp.940-946,Aug. 2006.

[18]D. K. Roumis, L. M. Frank. "Hippocampal sharp-wave ripples in waking and sleeping states." Current opinion in neurobiology, vol. 35, pp. 6-12, May. 2015.

[19] Vorhees CV, Williams MT. "Morris water maze: procedures for assessing spatial and related forms of learning and memory." Nat Protoc. vol. 1, no. 2, pp. 848-858.Jul. 2006.

[20] Al-Qazzaz NK, Bin Mohd Ali SH, Ahmad SA, Islam MS, Escudero J. "Selection of Mother Wavelet Functions for Multi-Channel EEG Signal Analysis during a Working Memory Task." Sensors (Basel). vol. 15, no. 11, pp. 29015-29035, Nov. 2015.

[21]J. J. Palop, L Mucke. "Network abnormalities and interneuron dysfunction in Alzheimer disease." Nat Rev Neurosci, vol. 17, no. 12, pp. 777-792. Nov 2016.

[22] T. F. Freund , I Katona "Perisomatic inhibition." Neuron, vol 56, no. 1, pp. 33-42. Oct 2007.

[23] Sanchez-Aguilera A, Quintanilla JP. "Sharp Wave Ripples in Alzheimer's Disease: In Search of Mechanisms." J Neurosci. 2021; vol. 41, no. 7, pp. 1366-1370, Feb. 2021.

[24]K. A. Pelkey, R Chittajallu, M. T. Craig,L Tricoire,J. C. Wester, and C. J. McBain, "Hippocampal GABAergic Inhibitory Interneurons.” Physiol Rev, vol. 97, no. 4, pp. 1619-1747. Oct 2017.

Contribution of individual authors to the creation of a scientific article (ghostwriting policy)

Duyan Geng writes this paper; Zeyu Gao conducted the experiment and analysis; Yan Wang optimized the experiment; Zhaoxu Qin reviews and edits it; Geng Pang and Ning Zhao proposed some suggestions. http://naun.org/main/format/contributor-role.pdf

\section{Creative Commons Attribution License 4.0 (Attribution 4.0 International, CC BY 4.0)}

This article is published under the terms of the Creative Commons Attribution License 4.0 https://creativecommons.org/licenses/by/4.0/deed.en_US 